\title{
Cloud Computing and Its Vision 2015!!
}

\author{
Anurag S. Barde
}

\begin{abstract}
Cloud computing" - "the cloud" is a familiar cliche for the internet, but when combined with "Computing", the meaning gets bigger and fuzzier. Being built on decades of research; Cloud Computing utilizes all recent achievements in virtualization, distributed and utility computing and networking. It implies a service oriented architecture through offering softwares and platforms as services, reduced information technology overhead for the end-user, great flexibility, and reduced total cost of ownership, subscription-based or pay-per-use services etc. This paper is a brief survey based of readings on "cloud computing" and it addresses the use of computing resources that are delivered as a service over a network, challenges ahead and possible applications along with its scope and use in future!!
\end{abstract}

Index Terms-Cloud computing, portability, security, risk, elasticity, scalability, deployment, virtualization.

\section{INTRODUCTION}

Cloud Computing is a general term used to describe a new class of network-based computing that takes place over the Internet, basically a step on from Utility Computing.

1) A collection or a group of integrated and networked hardware, software and internet infrastructure (called a platform).

2) Using the internet for communication and transport provides hardware, software and networking services to clients, to general public, enterprises, corporations and businesses markets.

3) These platforms hide the complexity and details of the underlying infrastructure from users and applications by providing very simple graphical interface or API (Applications Programming Interface).

4) In addition, the platform provides on demand services that are always $\mathrm{ON}$, anywhere, anytime and anyplace.

5) Pay as-you- use. It's elastic (Fig. 1).

6) Scale up and down in capacity and functionalities [1].

\section{What is the Cloud Computing?}

Cloud computing is receiving a great deal of attention, both in publications and among users, from individuals at home to all the governments. Yet, it is not always clearly defined. Cloud computing is a subscription-based service where you can obtain networked storage space and computer resources. One way to think of cloud computing is to consider your experience with email. Your computing is to consider your experience with email. Your email client, if it is Yahoo!, Gmail, Hotmail, and so on, takes care of housing

Manuscript received January 15, 2013; revised April 17, 2013.

Anurag S. Barde is with the Maharashtra Institute of Technology College of engineering, Pune, India (e-mail: anuchikku@ rocketmail.com). all of the hardware and software necessary to support your personal email account. When you want to access your email, you open your web browser, go to the email client, and log in. The most important part of the process is having internet access. Your email is not housed on your physical computer You access it through an internet connection and you can access it anywhere. If you are on a trip, at work, or down the street getting coffee, you can check your email as long as you have access to the internet. Your email is different than software installed on your computer, such as a word processing program. When you create a document using word processing software, that document stays on the device you used to make it unless you physically move it. An email client is similar to how cloud computing works. Except instead of accessing just your email, you can choose what information you have access to within the cloud [2].

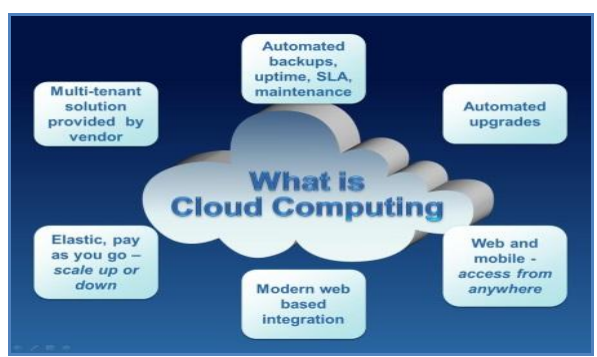

Fig. 1. What is cloud computing?

\section{GENERAL DiscUSSION}

\section{A. How Much Data}

1) Way back Machine has $2 \mathrm{~PB}+20 \mathrm{~TB} / \mathrm{mth}$ (2006)

2) "All words ever spoken by human beings" $\sim 5 \mathrm{~EB}$

3) CERN'S LHC (Large Hydrogen Collider, i.e, World's highest enery particle accelerator) will generate $15 \mathrm{~PB}$ a year (2008) (Fig. 2)

4) Google processes $20 \mathrm{~PB} /$ day (2008) (Fig. 3)



Fig. 2. World's highest-energy particle accelerator

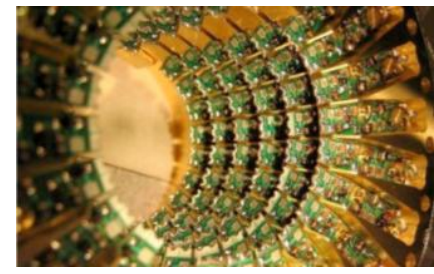

Fig. 3. Google's foray into faster computing. 


\section{B. Types of Clouds}

There are different types of clouds that you can subscribe to, depending on your needs. As a home user or small business owner, you will most likely use public cloud services.

1) Public Cloud - A public cloud can be accessed by any subscriber with an internet connection and access to the cloud space.

2) Private Cloud - A private cloud is established for a specific group or organization and limits access to just that group.

3) Community Cloud - A community cloud is shared among two or more organizations that have similar cloud requirements.

4) Hybrid Cloud - A hybrid cloud is essentially a combination of at least two clouds, where the clouds included are a mixture of public, private, or community cloud [3].

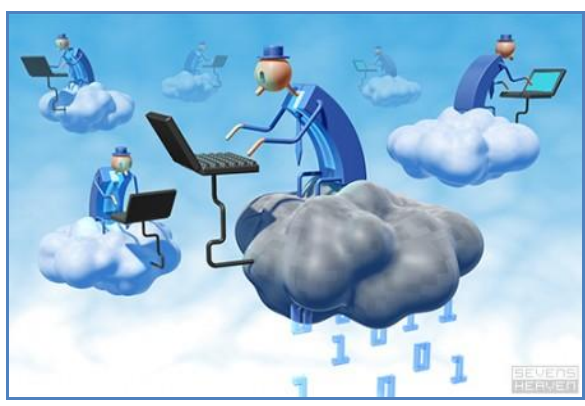

Fig. 4. World going crazy about cloud computing!

\section{Security}

The information housed on the cloud is often seen as valuable to individuals with malicious intent. There is a lot of personal information and potentially secured data that people store on their computers, and this information is now being transferred to the cloud. This makes it critical for anyone, to understand the security of the data and it is equally important to take personal precautions to secure the data. The first thing one must look into, is the security measures that your cloud provider already has in place.These vary from provider to provider and amongst the various types of clouds. What methods of protection do they have in place for the actual hardware that your data will be stored on? Will they have backups of your data? Do they have firewalls set up? If you have a community cloud, what are the barriers which keep your information separate from other companies? Many cloud providers have standard terms, a negotiation room in their cloud contract.A small business user may have slightly more room to discuss the terms of their contract with the provider and will be able to ask these questions during that time.There are many questions that you can ask, but it is important to choose a cloud provider that considers the security of your data as a major concern. No matter how careful you are with your personal data, by subscribing to the cloud, you will be giving up some control to an external source. This distance between you and the physical location of your data creates a barrier. It may also create more space for a third party to access your information. However, to take advantage of the benefits of the cloud, you will have to knowingly give up direct control of your data [4]. On the converse, keep in mind that most cloud providers will have a great deal of knowledge on how to keep your data safe. A Cloud provider possibly has more resources and expertise than the average user to secure their computers and networks. Whatever may be the security problems, but the fact is the world is going crazy for cloud computing usage (Fig. 4).

\section{Advantages and Disadvantages}

\section{1) Advantages}

Better performance: Due to the fact that no programs or files are loaded on the local PC, users will not experience delays when switching on/off their computers and also the internal network will be much faster since no internal traffic will occur.

Lower-cost computers for users: This point is one of the financial advantages of cloud computing. There is no need to purchase powerful and expensive equipment to use cloud computing since all the processing is not at your local computer but in the cloud. Since the application runs in the cloud, not on the desktop PC, that desktop PC does not need the processing power or hard disk space demanded by traditional desktop software [1].

Lower software costs: Using cloud computing there is no need to purchase software packages for each computer in the organization, only those employees actually using an application need access to that application in the cloud.

Increased power of computing: When using cloud computing, you can use the cloud computing power since you are no longer limited to what a single desktop computer can do.

Less IT infrastructure costs: The IT department of large organizations could experience decreasing on the expenses in regards with infrastructure with the adoption of the cloud computing technology. Instead of investing in larger numbers of more powerful servers, the IT staff can use the computing power of the cloud to supplement or replace internal computing resources.

Automatic software updates: All the software's need update and the great thing with cloud computing is that you do not have to worry for any updates and also your organization will not have any additional expenses when a new upgrade or update is necessary.

Less maintenance costs: Maintenance costs also will be reduced using cloud computing since both hardware and software maintenance for organizations of all sizes will be much less. For example, fewer servers are necessary in the organization which means that maintenance costs are immediately lowered. As to software maintenance, there is no software on the organization's computers for the IT staff to maintain.

Limitless storage capacity: The cloud offers virtually unlimited storage capacity.Also at any time you can expand your storage capacity with a small additional charge on your monthly fee.

Increased data safety: There is no point to worry for disk failures or a disaster at your office. All the data is stored in the cloud.

Anywhere access to your documents: When you are in the cloud, there is no need to take your documents with you. Instead, you can access your actual PC from anywhere, where 
there is Internet access available.

Latest version availability: One more thing in relation with documents is that when you edit one document at the office and then you go somewhere else and open it, the latest version will be displayed since all the work is done centrally in the cloud.

Use your computer from anywhere: This is one of the biggest advantages of cloud computing. Basically, when you use this technology, you are not limited to work on a single PC. You just use your "cloud PC" from anywhere and any PC and your existing applications and documents follow you through the cloud to you. Move to a portable device, and your applications and documents are still available.

\section{2) Disadvantages}

Internet connection is required: It is impossible to work if your Internet connection is down, since you are using Internet to connect to your "cloud PC". If there is no Internet connection, then no access.

Low-speed connections are not at all recommended: This is not a very important disadvantage since everybody today has at least $1 \mathrm{Mbps}$ connection at work and at home. However, it is important to mention that cloud computing cannot work with slow Internet connections such as dial-up since web-based applications often require a lot of bandwidth to download, as do large documents [1].

Sometimes is slow: Also, with fast connections, sometimes you might experience delays since web-based applications can sometimes be slower than accessing a similar software program on your desktop PC. The reasons for that are because of the demanding upload and download bandwidth that web applications need.

Your data is $100 \%$ in the cloud: All the data that you had until now on your local PC, it is stored in the cloud. Theoretically, data stored in the cloud is safe since a cloud hosting company uses several ways of backup in order ensure that, on any case the data will not be lost. However, if your data is missing (even one in a million), you have no physical or local backup of your data.

Stored data might not be secure: Data is stored "in the cloud". However, where exactly is the cloud and is it really secure? These are questions arising for users that have confidential data.

\section{E. Cloud Flavors}

1) Software as a service (SaaS)

2) Infrastructure as a Service (IaaS)

3) Platform as a Service (PaaS)

4) Data as a Service (DaaS)

5) Network as a Service (NaaS)

6) Storage as a Service (STaaS)

\section{F. Choosing a Cloud Provider}

Each provider serves a specific function, giving users more or less control over their cloud depending on the type. When you choose a provider, compare your needs to the cloud services available. Your cloud needs will vary depending on how you intend to use the space and resources associated with the cloud. If it will be for personal home use, you will need a different cloud type and provider than, if you will be using the cloud for business. The cloud provider will be pay-as-you-go,it means that if your technological needs change at any point, you can purchase more storage space (or less) from your cloud provider.

There are three types of cloud providers that you can subscribe to: Software as a Service (SaaS), Platform as a Service (PaaS), and Infrastructure as a Service (IaaS) [5]. There are three types which differ in the amount of control that one have over your information, and conversely, how much you can expect your provider to do for you. Briefly, here is what you can expect from each type.

1) Software as a Service - A SaaS provider gives subscribers access to both resources and applications. SaaS makes it unnecessary for you to have a physical copy of software to install on your devices. SaaS also makes it easier to have the same software on all of your devices at once by accessing it on the cloud. In a SaaS agreement, you have the least control over the cloud. Examples of providers using SaaS applications are facebook, salesforce.com etc. (Fig. 5)

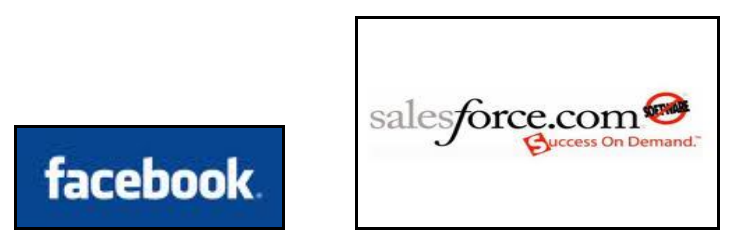

Fig. 5. Examples of providers using SaaS applications, i.e, facebook, salesforce.com etc.

2) Platform as a Service - A PaaS system goes a level above the Software as a Service setup. A PaaS provider gives subscribers access to the components that they require to develop and operate applications over the internet. Examples of providers using PaaS applications are Microsoft Azure, Google app engine etc. (Fig. 6)

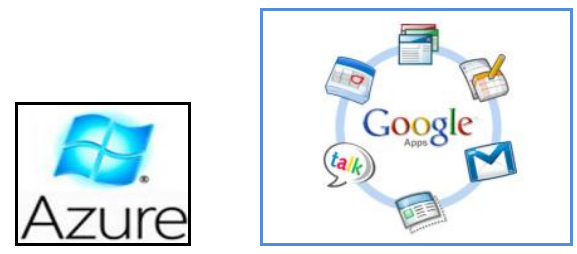

Fig. 6. Examples of providers using PaaS applications,i.e, Microsoft Azure, Google app engine etc.

3) Infrastructure as a Service - An IaaS agreement, as the name states, deals primarily with computational infrastructure. In an IaaS agreement, the subscriber completely outsources the storage and resources, such as hardware and software, which they need.Examples of providers using IaaS applications are terremark, Go Grid etc. (Fig. 7)

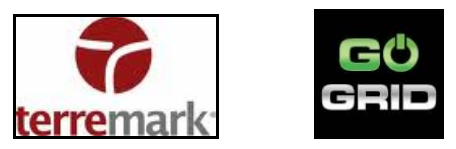

Fig. 7. Examples of providers using IaaS applications ,i.e, terremark, Go Grid etc.

As you go down the list from number one to number three, the subscriber gains more control over what they can do within the space of the cloud. The cloud provider has less control in an IaaS system than with SaaS agreement. 


\section{G. Future of Cloud Computing [6]}

\section{1) Current status of SaaS: SaaS (Best practices under development)}

1) There are several trade associations, promoting SaaS

2) There are several trade associations, promoting SaaS

3) There are many SaaS companies' e.g. Salesforce.com and NetSuite using Cloud infrastructure to increase scalability.

4) Google Apps and Zoho are SaaS competitors with MS Office.

5) Microsoft $\mathrm{SaaS}$ implementations (e.g. Exchange) available.

6) Traditional enterprise software dealers are moving to the Cloud (e.g. Oracle).

7) Interoperability between $\mathrm{SaaS}$ is possible using Web standards (possibly hosted in Cloud).

8) U.S. Government has created a SaaS Apps.gov Web site.

9) PaaS tools make it easier to create scalable, customized SaaS.

Predictions! There will be an explosive growth in SaaS applications due to low cost of entry for vendors and users, ability to rapidly deploy and update capabilities, elasticity and scalability of billing. The key issues will be:

1) Choosing appropriate applications for SaaS deployment.

2) Security, reliability, privacy, integration.

3) Avoiding lock-in to specific SaaS vendors.

4) Leading SaaS vendors (e.g. Facebook, salesforce.com) have or will attempt to become PaaS providers.

2) Current Status of PaaS, PaaS (Technology under development)

1) Google App Engine and Microsoft Azure are PaaS products integrating development and deployment.

2) Many smaller as well as major Cloud vendors e.g. Amazon, Salesforce.com are moving towards PaaS.

3) IaaS vendors will probably acquire or partner with PaaS suppliers e.g. VMware purchasing Spring Source. PaaS ecosystems will be a major arena of Cloud competition.

Predictions! PaaS will become the battleground for determining the future of Cloud Computing. All the major players will provide Cloud platform capabilities to attract a critical mass of applications. Several dominant Cloud ecosystems will emerge. The key issues will include:

1) Selection of appropriate PaaS providers.

2) Interoperability and portability across PaaS Clouds maintaining control of application lifecycle.

3) Future Differentiators for PaaS will be integrating with applications utilizing mobile devices and sensors.

\section{3) Current Status of Iaas: IaaS (Standards under} development)

1) Multiple companies are offering IaaS services for storage and computing e.g. Amazon, GoGrid, and Rackspace.

2) Virtualization management tools (e.g from Citrix and VMware) are offering IaaS.

3) U.S. Government has created an IaaS Cloud Storefront.

4) Without PaaS tools and utilities, IaaS can be considered a different business model for hosting.

Predictions! Core IaaS will become increasingly standardized and commoditized for horizontally scalable applications. There will still be diversity across multiple Cloud implementations (e.g. AWS and vCloud-based).Cross-Cloud tools and middleware will be available to enable interoperability and portability across core IaaS. The key issues include:

1) Integration with existing systems and data centers.

2) Reliability, security, auditing.

3) Management and governance of applications.

4) IaaS providers will increasingly add new utilities and PaaS capabilities as differentiators.

\section{Conclusions}

To summarize, the cloud computing provides many options for everyday users as well as for large and small businesses. It opens up the world of computing to a broader range of uses and increases the ease of use by giving access through any internet connection.

If you are considering using the cloud, be certain that you identify what information you will be putting out in the cloud? Who will have access to that information? What you will need to make sure it is protected? \& what is the reputation and responsibilities of the providers, consider everything before you sign up! However, with increased ease also come drawbacks. You have less control over, who has access to your information and little to no knowledge of where it is stored. You also must be aware of the security risks of having data stored on the cloud. The cloud is a big target for mischievous individuals and may have disadvantages because it can be accessed through an unsecured internet connection.

1) Cloud Computing is outperforming the IT industry as real business value can be realized by customers.

2) Proper planning and migration services are needed to ensure a successful implementation.

3) Cloud solutions are simple to obtain, don't require long term contracts and are easier to scale up and down as needed.

4) Public and Private Clouds can be set up together to leverage the best of both.

5) Third party monitoring services ensures, customers are getting the most out of their cloud environment.

6) Security abidance and Monitoring is achievable with careful planning and analysis.

\section{ACKNOWLEDGEMENT}

Author heartily thanks his father, Sanjay Barde, mother Anjali Barde and aunt Vrushali Tickle, whose encouragement, guidance and support, from the initial to the final level enabled him to develop an understanding of the subject. Lastly, he offers his regards and sincere thanks to all those who have supported him in any respect during the completion of the project.

\section{REFERENCES}

[1] T. Harris. Cloud computing - an overview. [Online]. Available: http://www.south.cattelecom.com/Technologies/CloudComputing/007 1626948_chap01.pdf 
[2] D. Burford. Cloud computing -A brief introduction. [Online]. Available: http://www.ladenterprizes.com/pdf/CloudComputing .pdf

[3] Cloud computing. [Online]. Available: http:// www.en.wikipedia.org/wiki/Cloud computing

[4] D. G. Feng et al., "Study on cloud computing security," Journal of Software, vol. 22, no. 1, pp. 71-83, 2011.

[5] C. Quan and Q. N. Deng, "Cloud computing and its key techniques," Journal of Computer Applications, vol. 29, no. 9, pp. 2565, 2009.

[6] K. Won, "Cloud computing: Today and tomorrow," Journal of Object Technology, vol. 8, no. 1, pp. 65-72, 2009.

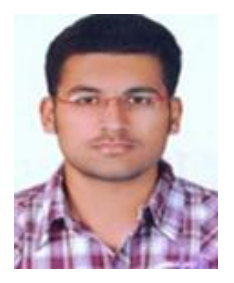

Anurag S. Barde was born in Bhandara, Maharashtra, India on November 18, 1992. Mr. Anurag Barde is currently a second year Engineering student at the Maharashtra Institute of Technology College of Engineering, Pune, India. Mr. Anurag Barde will be earning his Bachelor's degree in Mechanical Engineering in the year 2015. He is further interested in pursuing the graduate degree in robotics revolution using advanced computer vision to explore areas such as artificial intelligence, natural language and speech understanding, wireless networking, and nanotechnology.

Mr. Barde has been awarded with a certificate of excellence as a finalist in the robotics competition held in IIT KHARAGPUR, India. 